\title{
Incidence of hyponatraemia in the emergency unit
}

\author{
INGRID PRKAČIN ${ }^{1,2,}$ VALENTINA ĆORIĆ MARTINOVIĆ ${ }^{3}$, VESNA ĐERMANOVIĆ DOBROTA ${ }^{1}$, \\ ANA LEGOVIĆ ${ }^{1}$,BORNA VRHOVEC ${ }^{l}$, GORDANA CAVRIĆ ${ }^{1}$, PETRA DRAŽIĆ ${ }^{\prime}$ \\ ${ }^{1}$ Merkur University Hospital, Department of Internal Medicine, University of Zagreb, \\ School of Medicine, Zagreb, Croatia \\ ${ }^{2}$ Zagreb University School of Medicine, Zagreb, Croatia \\ ${ }^{3}$ General Hospital Vinkovci, Department of Internal Medicine, Vinkovci, Croatia
}

Corresponding author:

Ingrid Prkačin, Merkur University Hospital, Department of Internal Medicine, I. Zajca 19, 10000 Zagreb, Croatia

E-mail:ingrid.prkacin@gmail.com

\section{ABSTRACT}

Hyponatraemia is an electrolyte disorder, defined as a serum sodium concentration $(\mathrm{Na})<136 \mathrm{mmol} / \mathrm{L}$. It occurs in up to $30 \%$ of hospitalised patients. The purpose of this study was to evaluate the frequency of hyponatraemia among all patients during a one month period in the emergency unit. During the one month period in 2014, 570 patients were included in this study. The study was approved by local ethics committees and patients provided written informed consent.

Out of the 570 patients, 41 (7\%) had hyponatraemia. The median age was 67 $(65.02 \pm 14.09)$ years and the majority of the patients were men ( $\mathrm{M}: \mathrm{F}=23: 18$; 56.1:43.9\%). Mild hyponatraemia (serum $\mathrm{Na} 130-135 \mathrm{mmol} / \mathrm{L}$ ) was found in $71 \%$ (29/41), moderate (serum Na 125-129 $\mathrm{mmol} / \mathrm{L}$ ) in $17 \%(7 / 41)$, severe (serum Na $120-124 \mathrm{mmol} / \mathrm{L})$ in $5 \%(2 / 41)$, and extremely severe $($ serum $\mathrm{Na}<120 \mathrm{mmol} / \mathrm{L}$ ) in $7 \%(3 / 41)$ of patients. The treatment options included the restriction of fluid intake by administering hypertonic saline and loop diuretics.

We should be alert to acute hyponatraemia, especially in elderly patients with neurological manifestations and poor prognosis. The presented data are an important contribution to the better understanding of the epidemiology of hyponatraemia in Croatia.

Key words: hyponatraemia, emergency unit

\section{INTRODUCTION}

Hyponatraemia is the most common disorder of electrolyte balance. It occurs in up to $30 \%$ of hospitalised patients and leads to a wide spectrum of symptoms, some even life threatening. (1) It is associated with increases in both mortality and duration of hospital stay. Hyponatraemia is an electrolyte disorder defined as a serum sodium concentration $(\mathrm{Na})<136 \mathrm{mmol} / \mathrm{L}$ and is present on admission in $14.5 \%$ of all patients and in $15-20 \%$ of emergency hospital admissions. (1-3)

The early symptoms of hyponatraemia are vague and nonspecific, and may mimic the symptoms of a psychiatric disorder. The most common symptoms are nausea, drowsiness, headache, anorexia, and weight gain. These symptoms may be accompanied confusion, convulsions, coma and death. (2)

A group of patients with hyponatraemia is highly risky and hyponatraemia is recognized as a marker of increased risk of developing comorbidity and as a predictor of adverse outcome. (4-6)

The etiopathogenesis of hyponatraemia depends on the underlying disease or condition causing it. The most important factor in approaching a patient with hyponatraemia is an estimate of the volume status (distinguish between hypovolemia, hypervolemia and euvolemia). Euvolemia is predominantly caused by different drugs and inappropriate antidiuretic hormone secretion (SIADH), which is often not recognized. (3) Many conditions are associated with SIADH, and they can be classified into five main causes: neoplasia, hormonal disturbances, non-malignant lung disease (pneumonia, tuberculosis, asthma, abscesses), iatrogenic (due to taking a number of different drugs) and other (neurological diseases, prolonged physical activity, associated with AIDS, persistent vomiting, idiopathic in the elderly). (3) Hyponatraemia induced by drugs (antiinflammatory, diuretics, acetaminophen, antipsychotic, haloperidol, amitriptyline, fluoxetine) or tumours have incidence up to $30 \%$ (tumours of the lung, pancreas, thyroid). $(7,8)$

The management of patients with hyponatraemia remains very problematic.

There is a lack of clinical research regarding patients with hyponatraemia in emergency departments.

The purpose of this study was to evaluate the frequency of hyponatraemia among all patients during a one month period in an emergency unit.

\section{MATERIALS AND METHODS}

The aim of this study was to determine the incidence of hyponatraemia in patients examined in an emergency department's (ED) internal medicine unit during a onemonth period from December 1, through December 31, 2014. Five hundred and seventy patients from the Emergency Unit Internal Department were included.

Hyponatraemia is defined as a plasma sodium concentration $(\mathrm{Na})$ lower than 136 mmol per litre.

Patients with a reduced level of plasma sodium concentration were divided into four groups depending on the severity of hyponatraemia (3):

1) Group with mild hyponatraemia, defined as a plasma sodium concentration between 130-135 mmol per litre ( Na level of $130-135 \mathrm{mmol} / \mathrm{L}$ ),

2) Group with moderately severe hyponatraemia, defined as a plasma sodium concentration between 125-129 mmol per litre ( $\mathrm{Na}$ level of 125-129 mmol/L),

3) Group with severe hyponatraemia, defined as a plasma sodium concentration between 120-124 mmol per litre ( $\mathrm{Na}$ level of $120-124 \mathrm{mmol} / \mathrm{L}$ ),

4) Group with extremely severe hyponatraemia, defined as a plasma sodium concentration below $120 \mathrm{mmol}$ per litre 
(level $\mathrm{Na}<120 \mathrm{mmol} / \mathrm{L}$ ).

Most patients did not have a certain level of sodium in one-time urine (prior to infusion) or plasma/urine osmolality, so the same was not taken into analysis.

A chronic condition of low level of plasma sodium concentration (chronic hyponatraemia) was defined as a duration of more than 12 hours.

Acute hyponatraemia was defined as a duration of hyponatraemia of less than 12 hours.

We investigated the connection between the cause and severity of hyponatraemia with clinical outcomes of patients (improved, unchanged, death). Date were analyzed using SPSS16 software and CHIsquare test, considered statistically significant if $\mathrm{P}<0.05$. The study was approved by local ethic committees and patients provided written informed consent to participate.

\section{RESULTS}

The patients were an average age of 65.02 \pm 14.09 years, equal distribution between the sexes $(\mathrm{M} / \mathrm{F}=23 / 18 ; 56.1 \% / 43.9 \%)$, and $7 \%$ of patients had some form of hyponatraemia (41/570). Statistically there was more mild hyponatraemia (29/41; $71 \%)$ compared to the others (moderately severe $(7 / 41 ; 17 \%)$, severe $(2 / 41,5 \%)$ and extremely severe $(3 / 41 ; 7 \%)$ hyponatraemia) with $\mathrm{p}<0.05$. The majority of patients had a chronic condition of low levels of plasma sodium concentration (more than 12 hours) with symptoms of headache, nausea, vomiting and disorientation. The most frequent degree of hyponatraemia was mild (group 1) and most present in cardiac patients and iatrogenic caused by diuretic therapy $(11 / 29,38 \%)$, as compared to patients with malignant disease $(6 / 29$, $21 \%$ ), infectious conditions in patients with pulmonary and/or endocrinological diseases $(5 / 29,17 \%)$, in patients with uncontrolled diabetes $(3 / 29,10 \%)$, decompensated cirrhosis with complications $(3 / 29,10 \%)$, and due to bleeding from stomach ulcers $(1 / 29 ; 4 \%)$.

Moderate hyponatraemia (group 2) was most common in patients with disseminated malignant disease $(3 / 7,43 \%)$, as well as in patients with diabetes and hypertension treated with gentamicin $(1 / 3,33 \%)$ or a combination of furosemide and spironolactone $(2 / 3,67 \%)$. The most rare case was seen in a patient with chronic kidney dis- ease (CKD) with hypovolemia (1/7, 14\%). Severe hyponatraemia (group 3 ) was registered in patients with uncontrolled endocrine diseases (Addison, diabetes mellitus type 2) in the state of an infectious diseaseviral and/or bacterial (urosepsis, enterocolitis). Extremely severe hyponatraemia (group 4) was registered in cardiac patients with severe aortal stenosis and cardiomyopathies $(2 / 3,67 \%)$. In half of the patients with hyponatraemia improvement of the situation was achieved (22/41, 54\%). A third remained constant (14/41, 34\%), while $12 \%$ did not survive the disease (5/41).

Among patients who developed neurological complications, $80 \%$ did not survive (94\% had acute hyponatraemia). Patients with hyponatraemia in ED were treated with hypertonic solutions of $\mathrm{NaCl}$ (to raise serum Na 1-2 mmol/L/hour) along with an individual adaptation of the therapeutic approach. Hyponatraemia was not sufficiently treated in $80 \%$ patients during the later treatment.

\section{DISCUSSION}

Despite the well-known fact that hyponatraemia is associated with poor outcome and extended-stay treatment, many doctors are not aware of the importance of reduced levels of plasma sodium concentration. Hyponatraemia is an under-recognized problem in the emergency department (ED), and also insufficiently treated during the later treatment of patients. (3) Patients with hyponatraemia are an extremely heterogeneous group. (9-11) Early symptoms of hyponatraemia are vague and non-specific and can mimic the symptoms of neurological and psychiatric disorders, especially in case of acute severe hyponatraemia. (4) It is very important to know the duration of severe hyponatraemia. $(11,12)$ If it is within several hours specialists must consider conditions such as self-induced water intoxication associated with psychosis, and the use of 3,4-methylenedioxymethamphetamine (MDMA, or "ecstasy"). Clinical features include headache, delirium, vomiting, seizures, coma, neurogenic pulmonary edema, brain swelling with risk of fatal herniation. The goal of therapy is $100-\mathrm{ml}$ bolus of $3 \%$ saline three times as needed for severe symptoms (increase plasma sodium concentration by $4-6 \mathrm{mmol} /$ litre in the first 6 hours). If the duration of severe hyponatraemia is
1-2 days present are conditions such as postoperative hyponatraemia, especially in women and hyponatraemia associated with intracranial disease. Clinical features could be headache, delirium, vomiting, seizures, coma, neurogenic pulmonary edema, brain swelling with risk of fatal herniation. The therapy goal is $100-\mathrm{ml}$ bolus of $3 \%$ saline three times as needed for severe symptoms and an increase plasma sodium concentration by $4-6 \mathrm{mmol} /$ litre in the first 6 hours, but avoiding increasing plasma sodium concentration by $>10$ $\mathrm{mmol} /$ litre/day. (12)

If the duration of severe hyponatraemia is unknown or $\geq 2$ days, specialist must consider conditions associated with the high risk of the osmotic demyelination syndrome (plasma sodium concentration $105 \mathrm{mmol} /$ litre or less, hypokalemia, alcoholism, malnutrition, liver disease). Clinical features are malaise, fatigue, confusion, cramps, falls and seizures. The therapy goal is $100-\mathrm{ml}$ bolus of $3 \%$ saline to increase plasma sodium concentrations by 4-6 mmol/litre in the first 24 hours, but avoiding increasing plasma sodium concentration by $>8 \mathrm{mmol} /$ litre/day. $(11,12)$ Osmotic demyelination syndrome may develop when the plasma sodium concentration is increased rapidly in outpatients who became hyponatraemia while drinking normal amounts of water, as well as in hospitalized patients who became hyponatraemia over 2 or more days. (12) Our data showed that in patients with acute hyponatraemia (duration less than 12 hours) the levels of plasma sodium concentration (serum sodium) were very low, and these are the patients most often presented with stupor or coma or convulsions. Among the patients who developed neurological complications, $80 \%$ did not survive, of which $94 \%$ had acute hyponatraemia.

Hyponatraemia is usually a chronic condition of reduced levels of serum sodium with symptoms of headache, nausea, vomiting, disorientation. (12)

In this study we found that hyponatraemia is present most frequently in patients of older age and often overlooked. (9) Our own results show that the percentage of patients who were examined in the emergency department through the one month due to hyponatraemia is $7 \%$ of the total examined, lower than in the available literature data. (1) The most common was mild hyponatraemia, in patients with heart failure and iatrogenic caused by diuretic therapy (38\%), which is consistent with 
other studies. (5-7) The second most frequent were patients with malignant disease (21\%) and it is necessary to note that hyponatraemia may be the first and only sign of inappropriate secretion of antidiuretic hormone (SIADH). (8) Neurological complications, especially acute changes in motor skills and awareness, are an important factor in assessing outcomes. They indicate a high risk of death and require immediate reimbursement of hypertonic $\mathrm{NaCl}$ solution. (9)

Hyponatraemia must be corrected gradually with the use of fluid restriction and slow infusions of $3 \%$ saline, furosemide, or by treatment of the underlying causes, and in some cases with use of urea or vasopressin antagonists. (12)

\section{CONCLUSION}

In the ED caution should be exercised and the monitoring of patients with hyponatraemia should be strengthened, especially in patients with malignant diseases and chronic heart and renal disease. When the actual duration of hyponatraemia is unclear it should be presumed to be chronic. Chronic hyponatraemia should be corrected gradually with the use of fluid restriction and slow infusions of 3\% saline, furosemide, urea or vasopressin antagonists, or by treatment of the underlying cause.

We should be alert to acute hyponatraemia, especially in elderly patients with neurological manifestations.

The presented data are an important contribution to the better understanding of the epidemiology of hyponatraemia in the ED in Croatia.

\section{REFERENCES}

1. Upadhyay A, Jaber BL, Madias NE. Incidence and prevalence of hyponatremia. Am J Med 2006; 119: S30-S35.

2. Hoorn EJ, Zietse RA. Hyponatremia and mortality: how innocent is the bystander? Clin J Am Soc Nephrol 2011; 6: 951-953.

3. Thompson C, Berl T, Tejedor A et al. Differential diagnosis of hyponatraemia. Best Pract Res Clin Endocrinol Metab 2012; 26: S7-S15.

4. Kim DK, Joo KW. Hyponatremia in patients with neurologic disorders. Electrolyte Blood Press 2009; 7:51-57.

5. Schrier RW, Sharma S, Shchekochikhin D. Hyponatremia: more than just a marker of disease severity? Nat Rev Nephrol 2013; 9: 37-50.

6. Fillippatos TD, Elisaf MS. Hyponatremia in patients with heart failure. World J Cardiol 2013; 5: 317-328.

7. Liamis G, Milionis H, Elisaf M. A review of drug-induced hyponatremia. Am J Kidney Dis 2008; 52: 144-153.

8. Onitilo AA, Kio E, Doi SA. Tumor-related hyponatremia. Clin Med Res 2007; 5: 228-237.

9. Sharabi Y, Illan R, Kamari Y et al. Diuretic induced hyponatraemia in elderly hypertensive women. J Hum Hypertens 2002; 16: 631-635.

10. Soupart A, Coffernils M, Couturier B,Gankam-Kengne F, Decaux G. Efficacy and tolerance of urea compared with vaptans for long-term treatment of patients with SIADH. Clin J Am SocNephrol 2012;7: 742-747.

11. Sterns RH. Disorders of Plasma Sodium — Causes, Consequences, and Correction. N Engl J Med 2015; 372: 55-65. DOI: 10.1056/ NEJMra1404489.

12. Spasovski G, Vanholder R, Allolio B et al. Clinical practice guideline on diagnosis and treatment of hyponatraemia. Nephrol Dial Transplant 2014; 29: Suppl 2: i1-i39. 